\title{
Optimal Geometry of Holes in a Cable Tray for Offshore Plants
}

\author{
Daehoon Park ${ }^{1,2} \cdot$ Jangmyung Lee ${ }^{3, *}$
}

\begin{abstract}
This study analyzes the crosstalk effects caused by the geometry of holes in a cable tray in offshore plants. Using the analysis results, we determine the optimal hole geometry that can effectively reduce the tray weight under minimum crosstalk. It was previously shown that metal cable trays can reduce crosstalk among cables. However, the impact of hole geometry was not considered. This study demonstrates the impact of hole geometry on the crosstalk. In addition, an algorithm is proposed to determine the optimal geometry of holes in the cable tray. The simulation results validate the proposed algorithm and can be useful for the designers of cable trays with holes.
\end{abstract}

Key Words: Cable Tray, Crosstalk, Offshore Plant, Scattering Parameter, Shielding Effectiveness.

\section{INTRODUCTION}

Typically, several power and signal lines are installed in offshore plants. Electromagnetic fields (EMFs) produced by highvoltage power lines can affect living organisms; therefore, a guide for determining a safe distance has been suggested [1].

These EMFs generated from the power lines can also affect other cables, resulting in interference among cables, which is referred to as called crosstalk [2]. The shielding characteristics of a cable tray have been extensively studied so far [3]. $S$ parameters were obtained through modeling and simulation, and experiments were conducted to determine the differences in shielding effectiveness (SE) based on the width-to-height ratios of U-shaped cable trays, which are commonly used in the industry [3].

The holes in a cable tray make it easy to attach cables or other fixings. In addition, they can reduce the tray's weight and enable drainage and ventilation. However, an increased area of holes can cause interference between cables. To minimize crosstalk between cables, holes are designed to have an optimal geometry. In this study, the SE of various shapes and numbers of holes in cable trays were compared through simulations and experiments. Using the analysis results, an algorithm was proposed to determine the optimal geometry of holes in the cable tray for the desired area of holes.

The rest of this paper is organized as follows. The algorithm proposed to find the optimal geometry is presented and validated in Section II. The conclusions are presented in Section III.

\section{PROPOSED ALGORITHM TO FIND OPTIMAL}

\section{GEOMETRY}

This paper proposes an efficient algorithm to determine the optimal geometry of holes in the cable tray for the desired area

Manuscript received November 6, 2020 ; Revised December 30, 2020 ; Accepted March 12, 2021. (ID No. 20201106-180J)

${ }^{1}$ Division of Robotics Convergence, Pusan National University, Busan, Korea.

${ }^{2}$ Advanced Research Center, Korea Shipbuilding \& Offshore Engineering, Seoul, Korea.

${ }^{3}$ Department of Electronics Engineering, Pusan National University, Busan, Korea.

"Corresponding Author: Jangmyung Lee (e-mail: jmlee@pusan.ac.kr)

This is an Open-Access article distributed under the terms of the Creative Commons Attribution Non-Commercial License (http://creativecommons.org/licenses/by-nc/4.0) which permits unrestricted non-commercial use, distribution, and reproduction in any medium, provided the original work is properly cited.

(c) Copyright The Korean Institute of Electromagnetic Engineering and Science. 
of holes. The detailed process of the proposed algorithm is described in this section.

\section{Dataset Collection}

To construct a dataset for training the relationship between the hole geometry in the cable tray and cable crosstalk, the input and output variables of the dataset must be defined. We chose width, number of columns, and number of rows of holes as the input variables. The hole height was fixed at $100 \mathrm{~mm}$ because it had no significant effect on the cable crosstalk. The range of variables is listed in Table 1 , and the number of datasets was 1,323 .

To construct a database, the simulation results of the crosstalk analysis performed with High Frequency Simulation Software (HFSS) were used. The $S$-parameter of near-end coupling $\left(S_{21}\right)$ had different values depending on the frequency. In addition, $S_{21}$ exhibited a similar tendency in the simulations. Instead of using all $S_{21}$ values at all frequencies, the representative value was used as the output value of $S_{21}$ for the proposed algorithm. $S_{21}$ at the 1 $\mathrm{MHz}$ frequency is one of the values with similar tendency. Therefore, the difference in $S_{21}$ between cable trays with and without holes at the $1 \mathrm{MHz}$ frequency was used as the output value of the dataset.

The low output value of $S_{21}$ indicates a high SE. As shown in Fig. 1, the expected values of crosstalk at the rest point between two typical points can be obtained using the crosstalk simulation results at the typical points.

Table 1. Range of variables

\begin{tabular}{lc}
\hline \multicolumn{1}{c}{ Variable } & Range \\
\hline Width $(\mathrm{mm})$ & $10-30$ \\
Number of rows & $1-7$ \\
Number of columns & $1-9$ \\
\hline
\end{tabular}

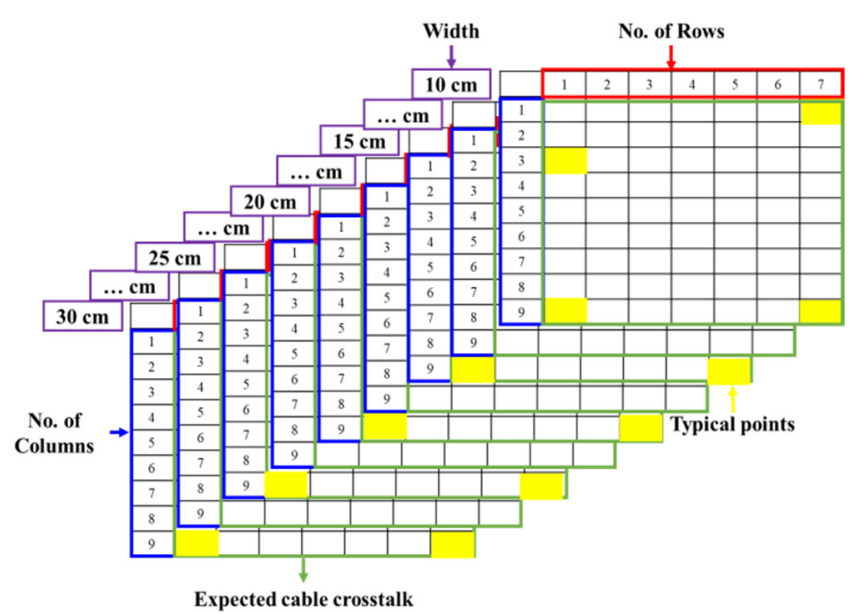

Fig. 1. Dataset configuration for training the relationship between the hole geometry in the cable tray and cable crosstalk.

\section{Training Dataset using Neural Network}

The dataset was trained using a neural network. The network had three inputs, 10 hidden layers, and one output and was trained using the Levenberg-Marquardt backpropagation method. This method is generally used for addressing data optimization problems [4]. For training, validation, and testing, the dataset was divided into three parts: $70 \%$ for training, $15 \%$ for validation, and $15 \%$ for testing.

\section{Finding Optimal Hole Geometry Using Neural Network}

In our proposed algorithm, when we enter the value of the desired area of the holes in the cable tray as input, the optimal geometry of holes with minimum expected crosstalk is suggested based on a trained neural network. The detailed algorithm is presented as follows:

Step 1: Input the desired area of holes and output possible cases of geometry with width, height, number of columns, and number of rows of holes.

Step 2: Output the predicted crosstalk based on the neural network by using possible cases of geometry obtained from the results of Step 1. The possible cases indicate possible hole geometries in the cable tray for the desired area.

Step 3: Find the hole geometry with minimum expected crosstalk from the results of Step 2.

\section{Experiments and Discussion}

We performed experiments to evaluate the performance of the proposed algorithm and to determine the optimal geometry of the holes in the cable tray. All approaches mentioned in this section were programmed using MATLAB. The training and testing processes were performed on a $\mathrm{PC}$ with an Intel i59400F processor, $32 \mathrm{~GB}$ RAM, and a clock rate of $2.9 \mathrm{GHz}$ using the Windows 10 operating system (64 bit).

For example, to design holes in cable trays with $84,000 \mathrm{~mm}^{2}$ area, we used an $84,000 \mathrm{~mm}^{2}$ area as input and then obtained the optimal hole geometry as the output using the proposed algorithm. As shown Table 2, when an $84,000 \mathrm{~mm}^{2}$ area of holes is used as the input, we can obtain the possible cases of various hole geometries, including the optimal geometry of holes.

To verify the experimental results obtained using the proposed algorithm to determine the optimal hole geometry, a simulation was performed using HFSS.

As shown in Fig. 2, the simulations were performed by varying the hole height as $13,15,24$, and $30 \mathrm{~mm}$ while maintaining the width at $100 \mathrm{~mm}$. Moreover, the number of holes was set as $9 \times 7 / 8 \times 7 / 5 \times 7 / 4 \times 7$ to maintain the total hole area.

As shown in Fig. 3, the simulation results were similar to those obtained using the proposed algorithm to find the optimal hole geometry using a neural network. 
Table 2. Experimental results pertaining to the height of parallel holes with similar area $\left(84,000 \mathrm{~mm}^{2}\right)$

\begin{tabular}{|c|c|c|c|c|}
\hline $\begin{array}{l}\text { Width } \\
(\mathrm{mm})\end{array}$ & $\begin{array}{l}\text { Height } \\
(\mathrm{mm})\end{array}$ & $\begin{array}{c}\text { No. of } \\
\text { columns }\end{array}$ & No. of rows & $\begin{array}{l}\text { Expected } \\
\text { crosstalk }\end{array}$ \\
\hline 30 & 100 & 4 & 7 & 8.14 \\
\hline 28 & 100 & 5 & 6 & 9.39 \\
\hline 24 & 100 & 5 & 7 & 7.79 \\
\hline 28 & 100 & 6 & 5 & 11.31 \\
\hline 23.33 & 100 & 6 & 6 & 9 \\
\hline 20 & 100 & 6 & 7 & 7.39 \\
\hline 30 & 100 & 7 & 4 & 14.51 \\
\hline 24 & 100 & 7 & 5 & 10.76 \\
\hline 20 & 100 & 7 & 6 & 8.37 \\
\hline 17.14 & 100 & 7 & 7 & 5.04 \\
\hline 26.25 & 100 & 8 & 4 & 13.73 \\
\hline 21 & 100 & 8 & 5 & 10.21 \\
\hline 17.5 & 100 & 8 & 6 & 6.22 \\
\hline 15 & 100 & 8 & 7 & 2.86 \\
\hline 23.33 & 100 & 9 & 4 & 13.1 \\
\hline 18.67 & 100 & 9 & 5 & 8.7 \\
\hline 15.56 & 100 & 9 & 6 & 3.91 \\
\hline 13.33 & 100 & 9 & 7 & 1.95 \\
\hline
\end{tabular}

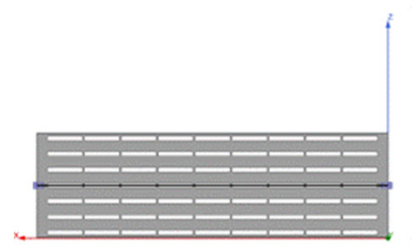

$13 \times 100 \times 9 \times 7$

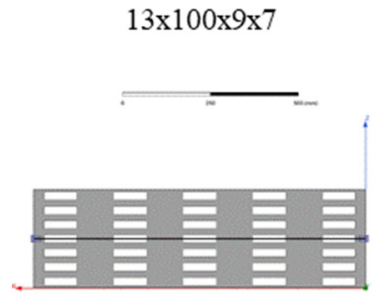

$24 \times 100 \times 5 \times 7$

Fig. 2. Cable tray modeling of parallel holes with different heights and similar area (approx. $84,000 \mathrm{~mm}^{2}$ ).

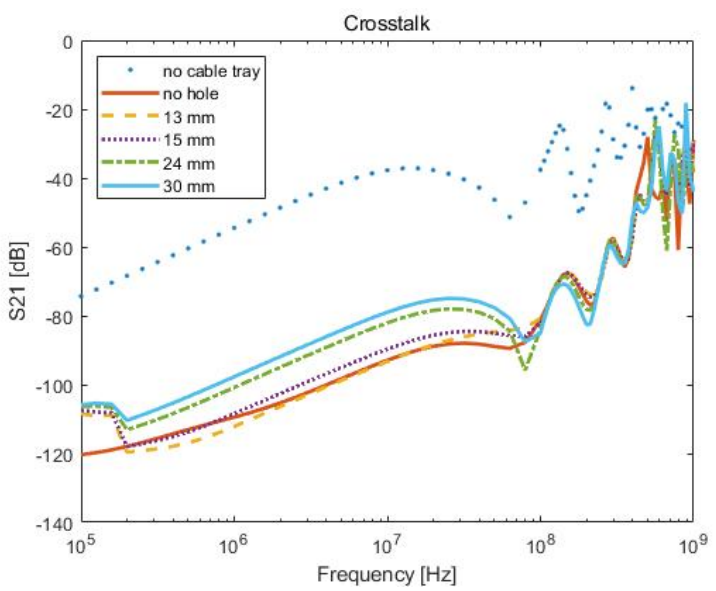

Fig. 3. Simulation results pertaining to the height of parallel holes with similar area $\left(84,000 \mathrm{~mm}^{2}\right)$.

\section{CONCLUSION}

The SE of cable trays used in offshore plants was analyzed for cable trays with and without holes through simulations and experiments. The $\mathrm{SE}$ of the parallel holes was verified by varying the width and length of the holes. Using the analysis results, an algorithm was proposed to determine the optimal geometry of holes in the cable tray and was verified through simulations. These results can be useful for the designers of cable trays with holes.

\section{REFERENCES}

[1] M. Nafar, "Health effects study and safe distance determination near an electrical distribution network," Journal of Electromagnetic Engineering and Science, vol. 19, no. 3, pp. 172-176, 2019.

[2] P. Xiao, P. A. Du, and B. Zhang, "An analytical method for radiated electromagnetic and shielding effectiveness of braided coaxial cable," IEEE Transactions on Electromagnetic Compatibility, vol. 61, no. 1, pp. 121-127, 2019.

[3] N. W. Ebertsohn, R. H. Geschke, and H. C. Reader, "Cable trays in EMC: Measurement and modeling to $30 \mathrm{MHz}$," IEEE Transactions on Electromagnetic Compatibility, vol. 49, no. 2, pp. 346-353, 2007.

[4] G. Hu, Z. Zhou, J. Cao, and H. Huang, "Non-linear calibration optimisation based on the Levenberg-Marquardt algorithm," IET Image Processing, vol. 14, no. 7, pp. 1402$1414,2020$. 